\title{
Nemencha (Le massif des) : Géographie
}

Jean-Louis Ballais

\section{(2) OpenEdition}

Journals

Édition électronique

URL : https://journals.openedition.org/encyclopedieberbere/2711

DOI : 10.4000/encyclopedieberbere. 2711

ISSN : 2262-7197

\section{Éditeur}

Peeters Publishers

\section{Édition imprimée}

Date de publication : 5 octobre 2012

Pagination : $5411-5427$

ISBN : 978-90-429-2718-6

ISSN : 1015-7344

\section{Référence électronique}

Jean-Louis Ballais, « Nemencha (Le massif des) : Géographie », Encyclopédie berbère [En ligne], 34 | 2012, document N37, mis en ligne le 15 décembre 2020, consulté le 17 février 2022. URL : http:// journals.openedition.org/encyclopedieberbere/2711; DOI : https://doi.org/10.4000/ encyclopedieberbere. 2711

Ce document a été généré automatiquement le 17 février 2022.

(c) Tous droits réservés 


\title{
Nemencha (Le massif des) : Géographie
}

\author{
Jean-Louis Ballais
}

1 À la différence du massif des Aurès*, leur célèbre voisin dans l'Atlas saharien algérien, les monts des Nemencha ont fait l'objet de peu d'études approfondies. Dans cette notice, on tentera de regrouper et de synthétiser les connaissances acquises en géographie, sans cacher les très nombreuses zones d'ombre qui persistent.

\section{Les premières études}

Les Nemencha semblent inconnus pendant la période antique et Idrîsî (1999, p. 130) ne les cite pas parmi les tribus berbères du Maghreb central. En revanche, pendant la période turque, la tribu des Nemencha, connue par Shaw (1980, p. 381-383) fait partie des tribus importantes de la région orientale du beylik de Constantine (Kaddache 1992, p. 149) et qui échappent à l'autorité beylicale. Cependant, le dictionnaire d'Ennery et Hirth (1840) les ignore totalement (de même que les Aurès, d'ailleurs). 


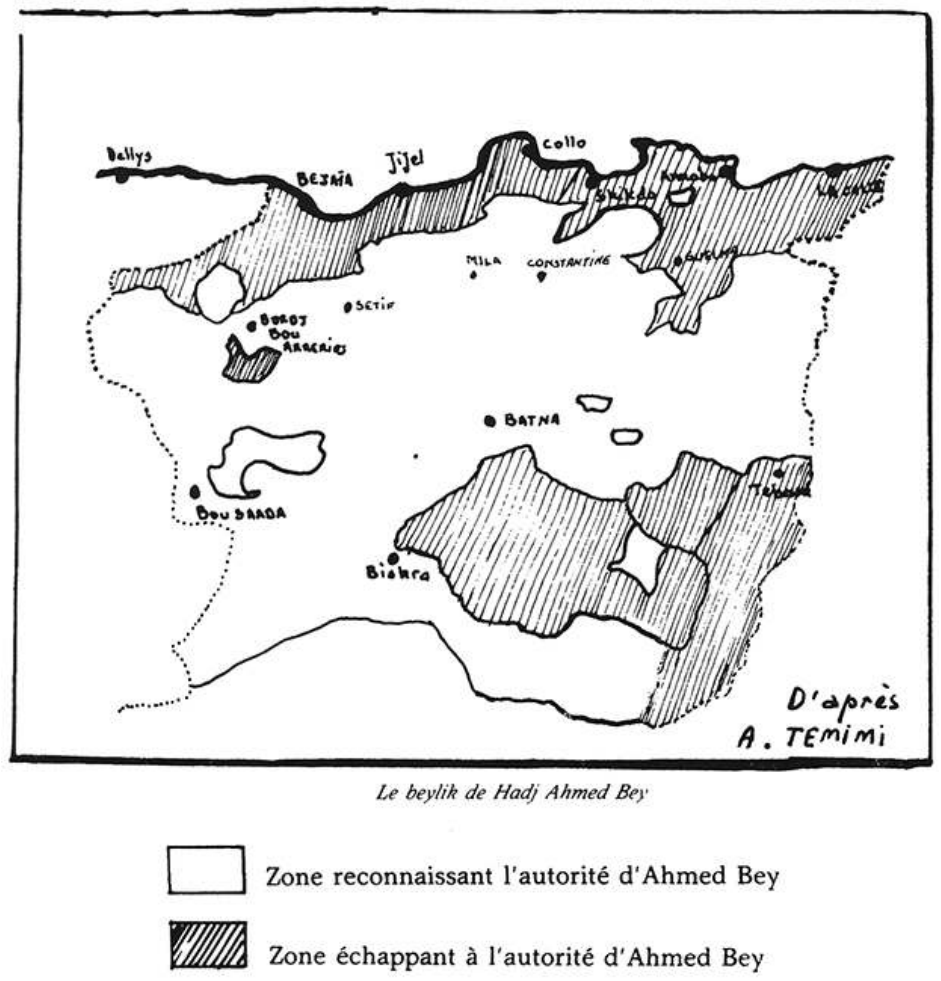

Fig. 1. Carte du beylik de constantine au début du XiX ${ }^{E}$ siècle.

3 La première étude est celle d'Émile Masqueray (1878), encore très partielle puisqu'elle se limite au djebel Chechar, entre les oueds el-Arab et Bidjer. Le premier, il insiste sur l'encaissement des vallées des oueds parallèles qui passent à l'aval, à la « chebka » qui précède la plaine saharienne. Le sens de "caillasse » qu'il attribue à Chechar convient bien à ce relief pierreux et aride en surface. Il observe le fonctionnement des versants calcaires de ces oueds et donne quelques indications sur la végétation et l'occupation $\mathrm{du}$ sol avec les cultures dans les vallées des oueds Bidjer et el-Arab principalement. Dans cette dernière, sont développées de petites oasis (Tboiou Ahmed, Ouldja, Chebla, Khiran) qui, comme dans celle de l'oued Bidjer au sud de Zawia cultivent surtout le palmier-dattier alors que le blé, l'orge, les oliviers, les figuiers et les abricotiers sont cultivés plus au nord. L'élevage nomade des moutons et des chèvres déplace les troupeaux des Nemencha (qui auraient été expulsés du djebel Chechar) des environs de Khenchela jusqu'au chott el-Djerid. Si d'anciens villages berbères comme le vieux Tizagrine ont été abandonnés, certains sont encore habités par de petits groupes (une trentaine de masures autour d'une koubba à Zawia, une centaine d'habitants au nouveau Tizagrine), la majorité de la population vit sous la tente (par exemple, 360 tentes de Beni-Barbar dans le Tafrent). Masqueray fait une place à part à la palmeraie (aux dattes renommées) et à la petite ville arabisée de Khanga Sidi Nadji, "centre d'action maraboutique autour duquel gravitent aussi bien les Amamra de l'Aurès que les Beni Mloul, les Beni Barbat, les Oulad Sullan, les Nememcha ». Khanga Sidi Nadji est d'ailleurs la seule vraie ville avec sa zaouia, sa mosquée, ses ateliers, ses boutiques, entourés par une petite muraille en terre à tours carrées.

Dans sa Nouvelle Géographie Universelle, É. Reclus (1886) s'appuie largement sur cette étude d'É. Masqueray. Tout en insistant sur le fait que les montagnes sont beaucoup moins explorées que l'Aurès, il distingue nettement le djebel Chechar $(1878 \mathrm{~m}$ au kef 
Ali-en-Nas), à l'est de l'oued el Arab, des montagnes des Nemencha (1 200 - $1400 \mathrm{~m}$ ) (p. 329-330). Ces deux ensembles paraissent être moins riches que l'Aurès en terres salubres et cultivables et, surtout, en ressources en eau. « Les peuplades de race zenata, Nemencha, Maafa, Achech, Beni-Barbar, Rechaïch ou Rechech, qui parcourent la région montagneuse comprise entre l'Aurès proprement dit et la frontière de la Tunisie, ont quelques villages fortifiés où ils déposent leurs céréales » (p. 537). Les infrastructures de transport manquent, les chemins ne sont praticables qu'à la belle saison. Il insiste (p. 543) sur le rôle commercial et potentiellement stratégique de Khanga Sidi Nadji dans la surveillance des Aurès et, surtout, sur le rôle des nombreuses zaouias, en particulier celle de Kheïrane «qui domine les populations du djebel Chechar et dont l'influence s'étend jusque sur les tribus de Tunisie.»

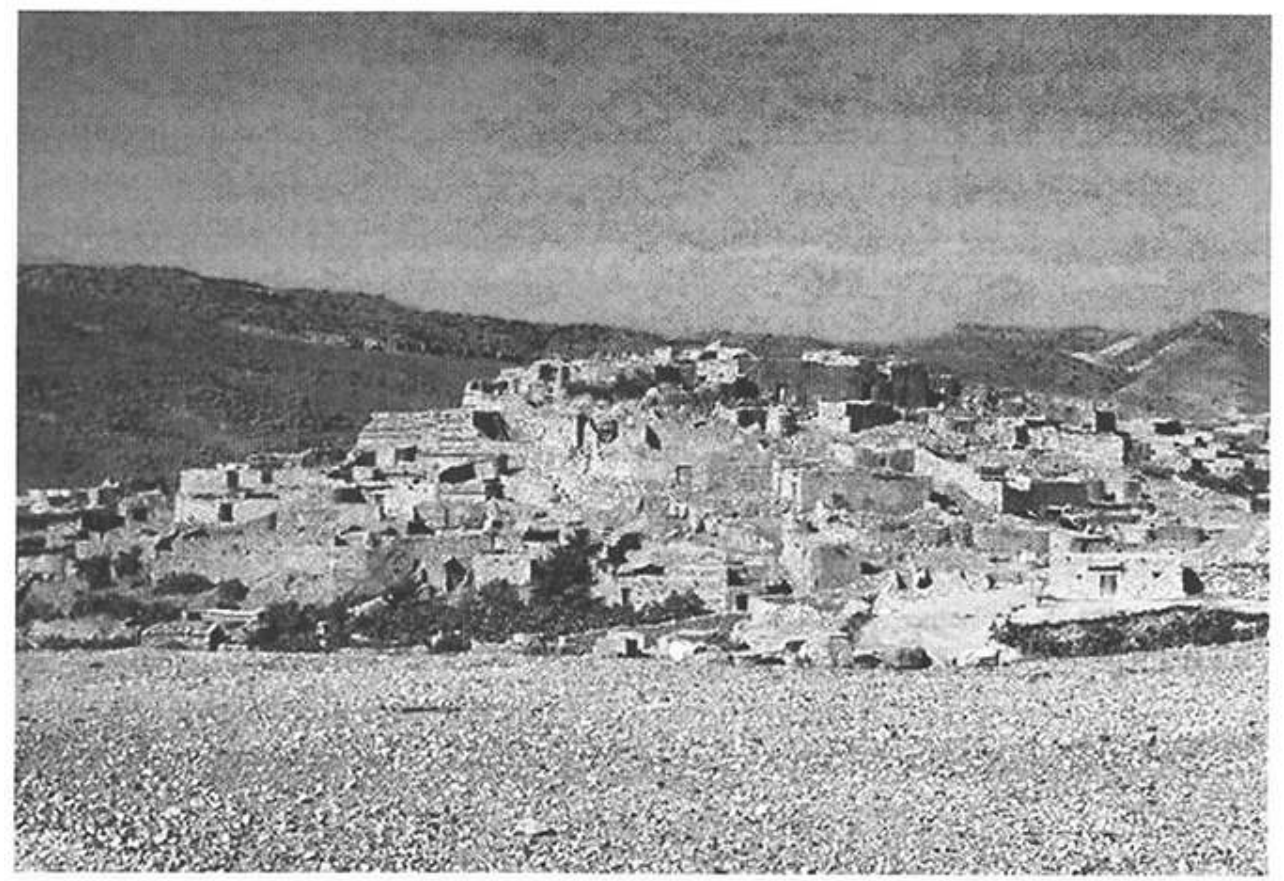

PHOTO 1. KHEIRANE EN 1978

Cliché J.-L. Ballais.

\section{La première monographie géographique}

5 Mais la première véritable monographie géographique des monts des Nemencha est celle de Blayac (1899), encore qu'elle s'intéresse principalement à la géographie physique. En prenant en compte la géologie nouvellement explorée, il est le premier à donner une description du relief structural : grande dépression synclinale des Ouled Rechaich, qu'il rattache encore à l'Aurès, immense plateau des Nemencha, ondulé au nord et presque tabulaire au sud, constitué de calcaires éocènes, qui plonge vers le Sahara et se prolonge loin vers la Tunisie, et, au nord, la région des dômes formée de trois grandes plaines elliptiques à une altitude de $1000 \mathrm{~m}$ en moyenne. Il décrit l'orientation NE-SW, identique à celle de l'Aurès, de ces plaines creusées dans les marnes du Crétacé supérieur, souvent marécageuses (Bahiret Telidjène, Outa Gassès), nettement limitées par des lignes continues de relief constituées de calcaires (souvent à 
silex et riches en phosphates déjà exploités plus au nord) de l'Éocène inférieur et qui comportent une butte centrale constituée de gypse, de dolomies et de marnes irisées.

Vers le sud, cuvettes et dômes perdent rapidement leurs caractères, le pendage des couches se rapproche de l'horizontale. Les calcaires à silex ne tardent pas à former une immense table vers $1000-1200 \mathrm{~m}$, faiblement inclinée vers le Sahara. Puis, brusquement ces calcaires se replient à angle droit pour disparaître sous les dépôts plus récents de la bordure saharienne. Pour Blayac, «la brusque inflexion des calcaires à silex donne au pays des Nemenchas une limite méridionale bien franche ». Cette limite montagneuse, orientée E-W, constituée par les djebels Ong et Sidi Abid, présente des abrupts infranchissables. Les oueds y ont creusé des canyons étroits et profonds dont les versants s'éboulent. Entre ces canyons, l'eau de pluie s'infiltre et va nourrir les nappes aquifères ; il existe des poches d'argile de décalcification (utilisées jadis par les Romains) et de rares dépressions fermées.

7 Malgré ces différences importantes entre le pays des dômes et le plateau, la végétation est très uniforme : tout le pays est déboisé, dépourvu d'arbres ou d'arbustes, «l'alfa à peu près seul couvre la plus grande partie des terres inutilisées par la culture. » À l'exception des rares tamarix et jujubiers qui survivent dans les gorges, il faut aller au nord de Chéria pour trouver des joncs, des roseaux et des massettes dans les marécages.

\section{Les recherches au $X X^{\mathrm{e}}$ siècle et au début du $\mathrm{XXI}$ siècle}

\section{Géomorphologie et géologie}

8 En fait, cette monographie reste la seule consacrée à l'ensemble des Monts des Nemencha. Les travaux postérieurs, peu nombreux et surtout consacrés à la géologie et à la géomorphologie, se sont intéressés soit à l'angle sud-ouest (Laffitte 1939; Ballais 1981), soit aux bordures nord-est (Kalla-Dridi 1994) ou est-sud-est (Chabin 1982, 1988), soit surtout à la région des dépressions (ou dômes) (Baghloul 1974; Lubell et al. 1975 ; Kalla 1995).

Ces recherches n'ont pas remis en cause la description géologique et géomorphologique de Blayac, mais elles l'ont précisée et nuancée. Le djebel Chechar (Laffitte 1939, carte hors-texte) est un val perché formé par la retombée orientale du dôme du djebel Toubount qui est la structure aurasienne la plus à l'est. Il est constitué par le synclinal d'Ounrhal formé principalement de calcaires éocènes sur les marnes du Crétacé supérieur et sur lesquels reposent, en discordance, des dépôts miocènes, au nord. Long, élevé, régulier, il constitue, sur la rive gauche de l'oued el Arab, une nette limite topographique qui le distingue des Aurès, bien qu'il ressemble aux grands vals de ce massif (Rhassira, Bouzina) avec cependant une différence majeure : il s'abaisse du sud vers le nord, vers les Hautes Plaines Constantinoises, à l'inverse de ceux des Aurès. Son crêt externe, formé principalement de calcaires londiniens sur des marnes épaisses, orienté vers l'ouest, est modelé, au sud, en mouvements de masse et coulées de solifluxion fossiles (Ballais 1981). Au pied de l'Ich Merzou, d'une indentation de la corniche sommitale sort une haute coulée boueuse qui a donné naissance à trois autres plus petites vers l'aval. Des pans de corniche glissés avec mouvement rotationnel s'observent également. En descendant vers Kheirane, des paquets de calcaires et de 
marnes éocènes, épais de 5 à $10 \mathrm{~m}$, ont recouvert les marnes sénoniennes verdâtres, entraînant des blocs de calcaire maestrichtien. Plus bas, on observe une grande coulée ancienne, à partir de laquelle s'est formée une petite coulée, également fossile, alors que fonctionnent actuellement, d'une part des décollements et, d'autre part, des ravinements actifs. Des dépôts hydromorphiques sombres se localisent dans les dépressions. Tout à l'aval, des coulées de solifluxion s'interstratifient dans la troisième terrasse alluviale de l'oued el Arab, très peu conservée, formée de petits galets. Plus au nord, au pied du djebel Frankou (1747 m), les marnes maestrichtiennes sont modelées par trois générations probables de coulées de solifluxion. En allant vers Babar, l'altitude et l'énergie du crêt externe décroissent, les coulées boueuses disparaissent, remplacées par le relief structural de corniches calcaires et de versants marneux ravinés, accidentés par de rares pans de corniche foirés.

La partie méridionale du val est affectée par un anticlinal d'orientation ouest-est, de l'ouest d'El Ouldja à Bou Yakadane, dissymétrique, avec un flanc sud qui plonge rapidement sous la Dekla-Chebka et un flanc nord qui passe au synclinal orienté SW-NE. C'est à cette charnière que se localisent les plus hauts sommets (1630 $\mathrm{m}$ au djebel Dra Bekhar et $1834 \mathrm{~m}$ à l'Ich Merzou), au nord de l'affleurement de marnes campaniennes qui forme une combe de flanc mal venue. L'anticlinal se continue vers l'est par une flexure (Birot, Dresch 1953) où les couches crétacées sont moins dégagées. Par suite d'un abaissement d'axe, elles disparaissent plus à l'est sous les couches éocènes, peu épaisses mais très étendues, affectées alors de grands chevrons irréguliers, mal venus (Guern el-Abiod : $1208 \mathrm{~m}$, djebel Tafrent : $1192 \mathrm{~m}$ ), dégagés entre l'entaille des oueds orientés du Nord au Sud, dont celle de l'oued Bidjer qui atteint également les marnes campaniennes.

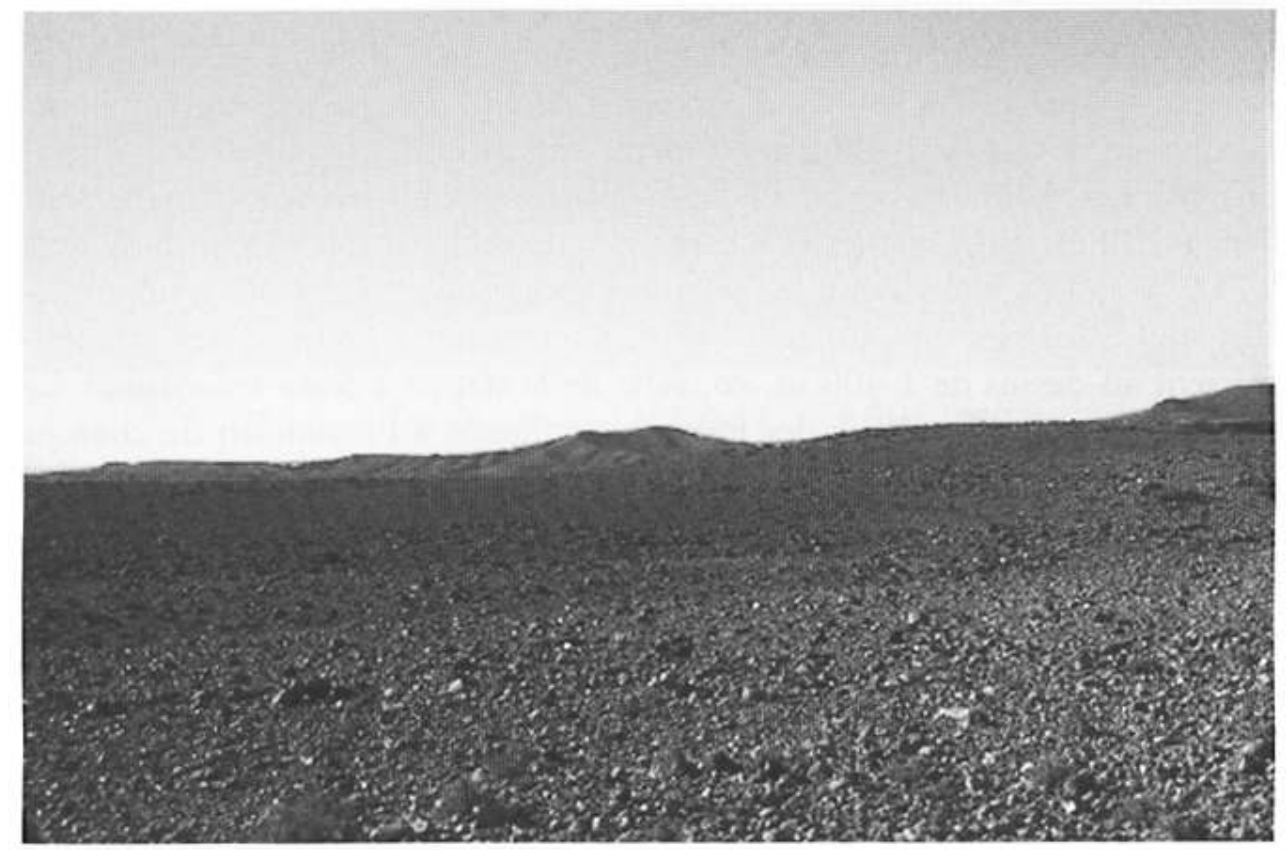

PHOTO 2. DJEBEL TIRIMBOU ET DEKLA-CHEBKA.

Cliché J.-L. Ballais, 1975

11 Sur le piémont sud, se succèdent, du sud au nord, les Guerguitt, la Dekla-Chebka et les Gueheb qui continuent vers l'Est les formes du pied des Aurès (Ballais 1981). Les Guerguitt, en bordure du Sahara, sont constitués par un monoclinal de poudingues 
pliocènes étroit, orienté WNW-ESE, à pendage vers le SSW, d'altitude 300-350 m, mais qui peut atteindre jusqu'à $591 \mathrm{~m}$ au dj. Halleb. Au nord, la Dekla-Chebka est creusée dans les marnes, sables et grès sableux du Miocène supérieur discordants sur les calcaires et marnes de l'Éocène inférieur. Cette dépression entoure les chaînons des Gueheb, chaînons étroits, d'orientation grossièrement WSW-ENE (photo 2), souvent faillés, qui dépassent $700 \mathrm{~m}$ au dj. Djermoun, et qui sont formés des calcaires et marnes du Lutétien et du Londinien. Ces formes disparaissent à l'est d'Aïn Mdila après avoir obliqué franchement vers le sud-est. De modestes accumulations d'hydrocarbures ont été découvertes à Guerguitt El Kihal nord et sud (Bergheul, 2005). La Dekla-Chebka est modelée en glacis dont la couverture porte parfois une croûte gypseuse (Khanga Sidi Nadji). Les silex des calcaires du djebel Tirimbou portent un cortex d'oxydes métalliques épais de 1 à $3 \mathrm{~mm}$. Sur les Guerguitt, restent accrochés des lambeaux de glacis, comme le glacis $V$ à croûte gypseuse de la cote 278 au sud du djebel Tirimbou

12 Á l'est du djebel Chechar, l'axe Bouffissane-Djarrar est une véritable barrière montagneuse qui s'allonge d'est en ouest sur près de $50 \mathrm{~km}$. C'est un plateau assez complexe, profondément disséqué par les oueds dont les canyons le recoupent perpendiculairement. Plus loin vers l'est, la barrière montagneuse perd de sa vigueur, les altitudes diminuent et le relief se morcèle. Un étagement peu être mis en évidence, sur le versant sud : jusqu'à $800 \mathrm{~m}$ d'altitude, un cortex d'oxyde métallique recouvre les silex éocènes et les ravinements sont nombreux dans les marnes. De 800 à $1000 \mathrm{~m}$, apparaissent les premières touffes d'Artemisia herba alba qui deviennent nombreuses au dessus de $1000 \mathrm{~m}$, en même temps que les ravinements disparaissent. Les vallons en berceau à dépôts hydromorphiques noirs apparaissent vers $1200 \mathrm{~m}$, ainsi que les sols caramel colonisés par des Euphorbes. Vers 1300-1350 m, s'observent les premiers éboulis, formés de blocs subarrondis, souvent dissous en surface. Enfin, les versants à peu près réglés apparaissent au-dessus de $1400 \mathrm{~m}$, couverts de la steppe à Stipa tenacissima. Le plateau est peu affecté par des formes karstiques, à l'exception de champs de lapiez très étendus, au-dessus de $1300 \mathrm{~m}$, surtout sur les calcaires à silex éocènes. Les dolines sont limitées au synclinal d'Ouled Rechaich où, dispersées, elles ne dépassent pas une profondeur de 3-4 $\mathrm{m}$ et un diamètre de quelques dizaines de mètres.

Au nord-nord-est s'étend la région des dépressions, cinq dépressions orientées SW-NE, limitées par des reliefs de calcaires éocènes, qui forment le contact entre les Hautes Plaines Constantinoises et le plateau des Nemencha (Lubell et al. 1975) : dépression d'Ounrhal, dépression d'Outa Guert-Outa Gassès, dépression d'Outa Zora-Outa Guibeur, dépression de Chéria (photo 4), dépression de bahiret Telidjène (photo 5).

14 L'amplitude des plissements qui ont engendré ces structures semble diminuer d'ouest en est; en effet, à l'ample structure synclinale d'Ouled Rechaich succède la structure anticlinale de Guessès déjà moins ample. Les anticlinaux de Hamimet Guibeur et de Hamimet Souda, plus à l'est, sont beaucoup plus modestes : $34 \mathrm{~km}$ de long et à peine $14 \mathrm{~km}$ de large, contre une centaine de kilomètres de long pour le synclinal d'Ouled Rechaich. Entre ces différentes structures anticlinales, s'intercalent d'étroites gouttières synclinales aux flancs parfois subverticaux (celui entre Outa Zora et bahiret Telidjène n'a que $15 \mathrm{~km}$ de large (Kalla 1995, p. 33-34)). La grande dépression synclinale complexe de Chéria leur succède vers le nord-nord-est.

Dans le centre des combes, formant colline, affleurent parfois des dépôts triasiques (marnes blanches ou rouges, gypse, dolomies noires, cargneules jaunes (Hamimet 
Souda, Hamimet Guibeur). Il s'agirait de pseudo-diapirs localisés sur des accidents NWSE et ouest-est (photo 3).

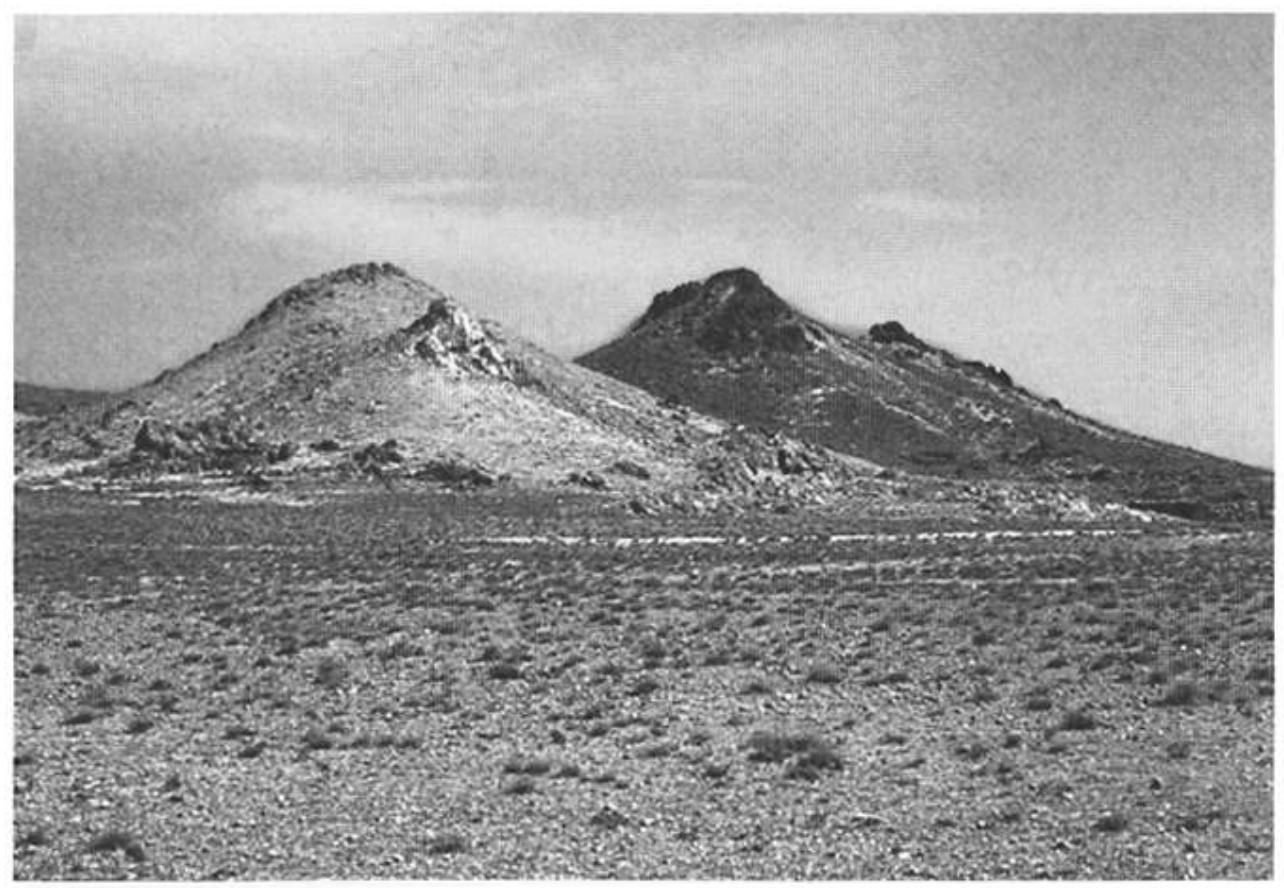

PHOTO 3. PSEUDO-DIAPIRS.

Cliché J.-L. Ballais, 1973

Depuis le Trias, prévalait un régime distensif qui avait permis une sédimentation essentiellement marine avec un grand développement de plates formes carbonatées au Jurassique et au Crétacé. L'inversion tectonique de la fin de l'Éocène, suivie des déformations d'âge Oligo-Miocène et post-Pliocène, s'est traduite par une intense structuration, des ablations et de nombreuses discordances d'importances variables (Bergheul, 2005). Les structures plissées orientées SW-NE ont été mises en place lors de la phase atlasique majeure de l'Éocène supérieur. Les structures orientées W-E seraient antérieures (Kalla 1995, p. 38-39).

Une surface d'aplanissement indiquée par la régularité de l'altitude des djebels (Lubell et al. 1975) daterait de l'oligocène (Kalla 1995), comme dans les Aurès voisins (Ballais 1981).

Des dépôts miocènes deltaïques s'accumulent ensuite localement, vers Aïn Babouche (30 $\mathrm{m}$ de sables) par exemple, au sud de Chéria, ou encore à Fridjou (au nord de Taberdga) $104 \mathrm{~m}$ de sables avec conglomérat et argile à Ostrea crassissima et pectinidés à la base (Laffitte 1939). En l'absence de fossiles, la limite avec des conglomérats supérieurs attribués au Pliocène reste discutable. 


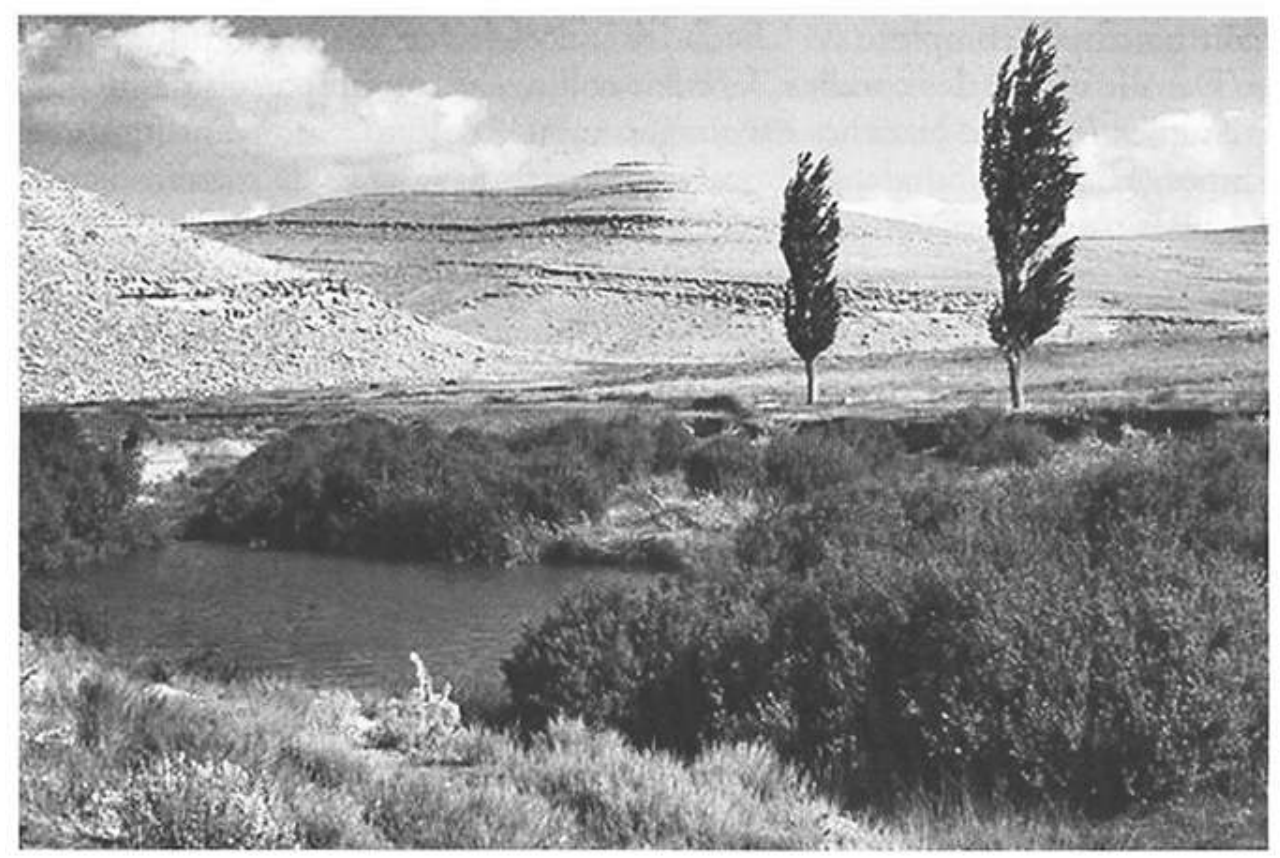

PHOTO 4. AÏN BABOUCHE.

Cliché J.-L. Ballais, 1973.

19 Au cours du Pliocène, le massif des Nemencha s'est comporté comme un horst qui s'est élevé rapidement entre deux zones qui s'abaissaient au nord et surtout au sud. Cette surrection post-miocène a été accompagnée de plissements d'orientation est-ouest bien développés sur le piémont méridional où ils constituent les Gueheb et les Guerguitt (et, plus à l'Est, la chaîne de Gafsa - Négrine*), mais très faibles dans le massif (quelques axes anticlinaux est-ouest dans la région de Chéria et dans la terminaison Sud de Hamimet Guerra et certains replis dans le synclinal d'Ouled Rechaich (Kalla 1995)). Le soulèvement post-néogène n'atteint que $1000 \mathrm{~m}$, la moitié de celui des Aurès. Cependant, l'âge du grand anticlinal est-ouest et de la flexure qui fait plonger le plateau sous la Dekla-Chebka demeure imprécis. Sur le piémont méridional, le plissement s'accompagne de grands accidents de type décrochant avec une composante chevauchante (vers Négrine), ou de type chevauchant avec une composante décrochante plus à l'ouest (Amia et al., 2005).

$\mathrm{Au}$ Quaternaire, cinq glacis se forment successivement, surtout bien développés dans la partie méridionale du djebel Chechar (Kalla 1995, p. 138-204) où l'entaille des oueds est la plus forte : le glacis VI qui peut dépasser $3 \mathrm{~km}$ de long, le glacis $\mathrm{V}$, présent également sur les piémonts et dans la dépression de Chéria, le glacis IV et surtout le glacis III qui occupe une grande place dans le paysage, particulièrement dans les dépressions et les piémonts. C'est un glacis d'ablation et souvent un glacis-terrasse. Il a pu éliminer les glacis antérieurs comme dans la bahiret Telidjène (photo 5). Il est le dernier à avoir enregistré les effets de la tectonique quaternaire (djebel Radama) (Lubell et al. 1975). Enfin, le glacis II, très fréquent, mais de superficie réduite. Dans les dépressions d'amont, très souvent, en raison de la faible entaille des oueds, les glacis ne sont pas étagés et leurs couvertures se superposent (nord-est de la bahiret Telidjène, Chéria). Plus généralement, les étagements se simplifient et se réduisent de l'ouest vers l'est. Le raccord entre les versants réglés des reliefs qui limitent les dépressions et les 
couvertures de glacis, parfois cryoturbées (glacis II du djebel Radama) à encroûtement ou croûte calcaire fréquents, se fait par des dépôts lités.

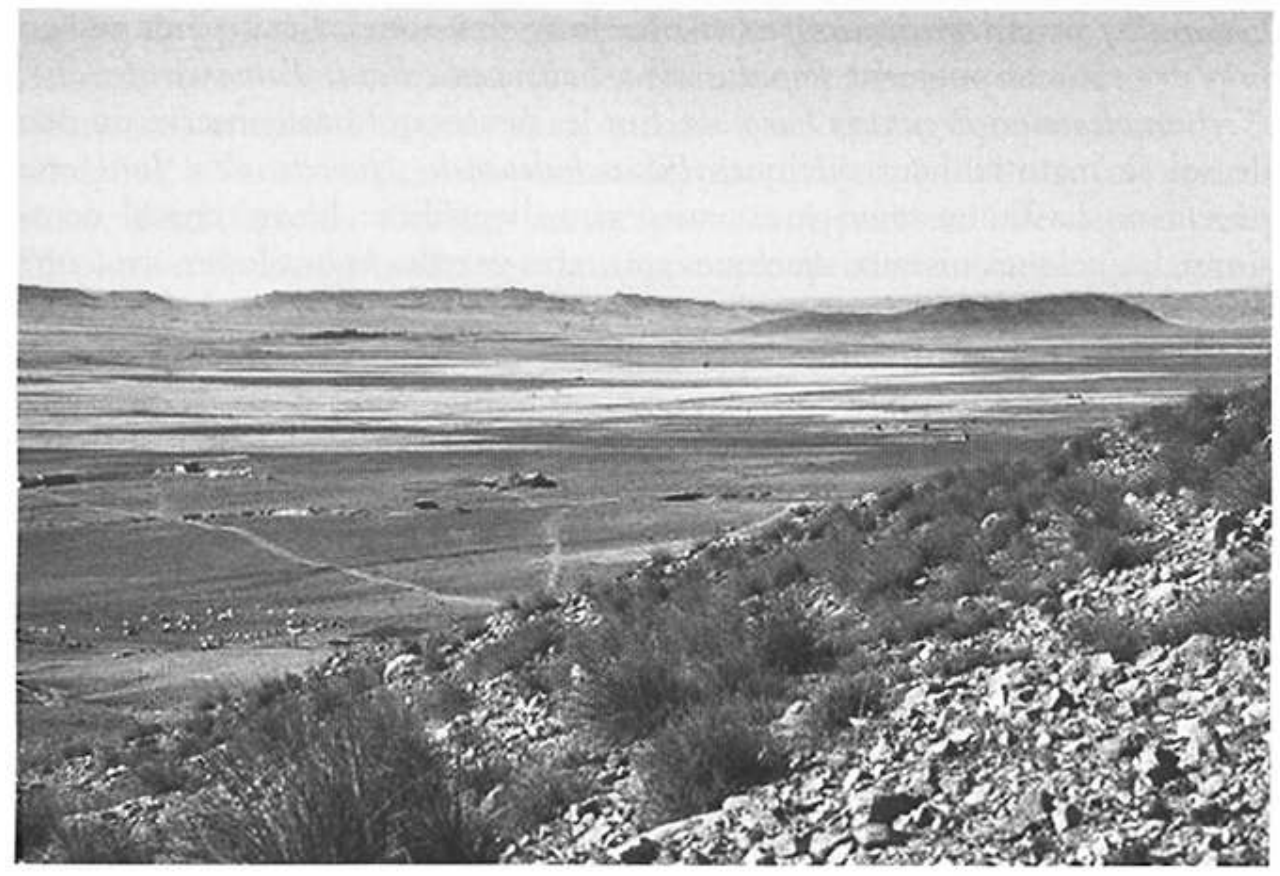

PHOTO 5. BAHIRET TÉLIDJÈnE.

Cliché J.-L. Ballais, 1973

\section{Le problème de l'absence de forêts}

L'absence ou, du moins, la rareté de la végétation arborée a été remarquée précocement (Masqueray 1878; Blayac 1899) et pose un problème environnemental majeur, à proximité des grandes forêts de Cedrus atlantica et de Pinus halepensis des Aurès (Ballais 1989). Á la fin du XIX ${ }^{\text {e }}$ siècle, Masqueray décrit une steppe à Stipa tenacissima et Artemisia herba alba, les lits des oueds étant colonisés par Tamarix et Nerium oleander (Blayac y ajoute Ziziphus lotus). La faune est, elle aussi, très pauvre: mouflons et gazelles transhumants, vautours blancs, aigles et milans chasseurs de goundis, quelques pigeons. Les observateurs suivants y ajoutent quelques Pinus halepensis, Juniperus phoenicea (Despois et Raynal 1967) et Juniperus oxycedrus (Birot, Dresch 1953). Les observations plus systématiques de Kalla (1995, p. 70) confirment l'absence de forêt: la steppe occupe les trois quarts de la surface et monte jusqu'à $1600 \mathrm{~m}$. Le matorral à Juniperus phoenicea, Quercus ilex et Pinus halepensis arbustif, avec une strate dense de Stipa tenacissima et de Rosmarinus officinalis, se limite au djebel Chechar sur lequel pousse également un peuplement lâche de Fraxinus xanthoxyloides adultes et de très rares Juniperus oxycedrus et Juniperus thurifera, eux aussi adultes ainsi que quelques Crataegus à orientation Nord (Ballais 1981). Lubell et al. (1975) observent que la végétation actuelle de la région des dépressions consiste en une steppe dégradée composée surtout d'herbacées et de buissons comme Stipa tenacissima et les deux armoises (Artemisia herba-alba et Artemisia campestris). Le long des cours d'eau pérennes ou près des sources poussent Populus alba, Salix pedicellata, Tamarix africana, Nerium oleander, Peganum harmala. Sur les pentes qui ont conservé un peu de sol se maintiennent quelques Pinus halepensis, Quercus ilex, Juniperus phoenicea. La faune 
sauvage est aussi peu diversifiée : lièvre, chacal occasionnel, quelques oiseaux, quelques très rares gazelles et antilopes, avec une microfaune de gerbilles, scorpions et escargots terrestres.

Or, il ne fait aucun doute que le climat des Monts des Nemencha est semi-aride à hiver froid, donc favorable à l'arbre, avec des précipitations moyennes annuelles de $256,7 \mathrm{~mm}$ à Chéria et $284 \mathrm{~mm}$ à Babar (Kalla 1995). Elles atteindraient, sur les plus hauts sommets, $550 \mathrm{~mm}$ au nord et 350 à $450 \mathrm{~mm}$ au sud-ouest. La neige tombe 7 à $8 \mathrm{j} / \mathrm{an}$ dans les dépressions ( 2 à 4 jours à Chéria), $20 \mathrm{j} / \mathrm{an}$ dans le val des Ouled Rechaich où l'épaisseur atteint fréquemment $30-35 \mathrm{~cm}$. Le Houérou et al. (1979) confirment que l'isohyète $300 \mathrm{~mm}$, au pied sud des Nemencha, et qui se localise vers $1100-1200 \mathrm{~m}$, marque la limite inférieure des zones où les espèces végétales utilisées traditionnellement pour les reboisements (comme Pinus halepensis) ont de réelles chances de reprises. Enfin, il faut rappeler que l'alfa est une plante forestière des forêts claires qui tend à occuper la place laissée vide par la disparition des ligneux (Djebaili 1984, p. 64).

Il apparait donc clairement que l'absence de forêt ne peut pas résulter de facteurs naturels seuls. Pour J.-P. Chabin (1988), la période de la deuxième moitié du Moyen-Âge au XIX ${ }^{\mathrm{e}}$ siècle porte peu de responsabilité dans cette absence car la société araboberbère était relativement peu prédatrice, ce que montre la survivance des grands fauves (lions, panthères) et le fait qu'il existait encore des boisements très clairs sur les collines du Petit-Sahara au XIX ${ }^{e}$ siècle. Pour Blayac (1899), si le "vrai pillage » exercé par les populations en quête de combustible sur les rares arbres survivants dans les gorges joue un rôle, il a remarqué «bien souvent dans la province de Constantine que les calcaires à silex de l'Eocène inférieur qui couvrent ici la plus grande étendue du plateau et de la région des dômes sont toujours dénudés... Ces calcaires fournissent très peu de terre de décalcification ; peut-être est-ce une des causes de leur aridité. »

Les analyses palynologiques effectuées à l'occasion de recherches préhistoriques (Lubell et al. 1975) ont fourni peu de résultats très solides en raison du grand nombre de grains d'espèces inconnues ou indéterminées. Elles ont cependant mis en évidence, dans le membre 2 de la basse terrasse alluviale de l'oued Chéria-Mezeraa (tête de l'oued Hallail) au pied du djebel Mezeraa la présence de Salix et Cedrus accompagnés ponctuellement d'Acacia et de Pinus vers 5839 +/- 95 B.P. Des macrorestes de Quercus ilex et Cupressus sp. étaient également présents. Dans les dépôts de la même terrasse alluviale de l'oued Chéria-Mezeraa (membre 4), juste en dessous d'un canal d'irrigation antique, des pollens d'Acacia, de Cedrus et de Quercus ilex s'observent constamment, accompagnés ponctuellement de Pistacia et Pinus. Même si les pollens de plantes steppiques sont dominants, cette variété de pollens d'arbres dont certains ont disparu du massif (Cedrus et Acacia) montre une importance des formations arborées nettement supérieure à factuelle. On peut donc penser que, même compte tenu de la tendance à l'aridification du climat depuis environ 4000 ans (cf. ci-dessous), deux facteurs d'origine anthropique ont joué un rôle capital dans l'évolution de la végétation vers la steppe actuelle: la consommation considérable de ligneux pendant la période capsienne et la mise en culture pendant la période romaine. Si la première reste mal connue, la deuxième est marquée, dans les premiers siècles de notre ère, par une forte érosion des sols fragilisés par des siècles de labours. Á l'occasion de courtes phases de pluies intenses (Ballais 1995 ; Ballais et al. 2003), ces sols ont subi une forte ablation et se 
sont accumulés dans le fond des vallées (Ballais 1976). Ainsi, les arbres ont été privés du substrat indispensable à leur développement et à leur reproduction.

\section{L'évolution morphoclimatique holocène}

La collaboration entre préhistoriens et géographes a été particulièrement fructueuse dans les Nemencha car elle a permis l'élaboration de la première chronologie morphoclimatique de l'Holocène du Maghreb central et oriental (Lubell et al. 1975; Ballais 1976; Farrand et al. 1982). Des travaux ultérieurs sur les Nemencha (Ballais et Benazzouz 1994) et dans des régions proches (Ballais 1991; Benazzouz, 2000) ont permis son enrichissement, si bien qu'on peut synthétiser l'évolution de la façon suivante. L'extrême fin du Pléistocène supérieur, le Tardiglaciaire, se marque par au moins une pulsation humide génératrice de pluies locales qui provoquent le redémarrage des ruissellements et l'accumulation de la terrasse alluviale II dans les Nemencha dès avant 11879 +/- 286 B.P. (Farrand et al. 1982 ; Ballais et Benazzouz 1994).

$\mathrm{Au}$ Maghreb oriental (Ballais et al., 2003), l'Holocène inférieur se caractérise par une augmentation de l'humidité qui provoque l'accumulation de la base de la basse terrasse alluviale (terrasse alluviale I) de l'oued Chéria-Mezeraa. Une phase aride l'interrompt vers 7500 - $7000 \mathrm{BP}$, mais, dans l'étage semi-aride, elle reste hautement hypothétique puisque, par exemple, l'oued Redif accumule toujours des dépôts fins vers 7340 +/- 115 BP. L'optimum climatique (?) du milieu de l'Holocène (7000 - 3000 BP ?) permet ensuite la stabilisation des versants et le développement de la pédogénèse : dans les Nemencha, se forment alors des sols bruns sur les versants et des sols vertiques dans les dépressions mal drainées. À la différence des écoulements qui ont créé la basse terrasse alluviale, ces sols supposent des géosystèmes en équilibre, la stabilité de la surface topographique à long terme permettant le développement d'une végétation relativement dense, une forêt claire méditerranéenne avec notamment Cedrus en altitude (Couvert 1972). Cependant, l'ablation des sols et des escargotières capsiennes continue et alimente l'accumulation de la terrasse alluviale. Donc, la répartition saisonnière des précipitations était meilleure que pendant l'Holocène inférieur et l'intensité des pluies plus faible. De nombreux oueds deviennent alors des rivières pérennes. L'Holocène récent préhistorique est une période encore mal connue et mal datée. Elle est caractérisée par l'aridification progressive du climat: son commencement est traditionnellement placé vers $4500-4000$ BP, ce qui est vrai pour la grotte Capéletti, dans les Aurès, mais l'oued Chéria-Mezeraa continue alors à accumuler sa basse terrasse alluviale. Cependant, localement, à altitude moyenne, les oueds Chéria-Mezeraa et Redif, moins bien alimentés à cause de l'aridité, entaillent un chenal dans la basse terrasse alluviale, plus tard comblé par des galets qui débordent sur cette basse terrasse alluviale. Simultanément, la fragmentation mécanique réapparaît au-dessus de $1700 \mathrm{~m}$ : de minces pellicules d'éboulis à matrice brune couvrent les éboulis plus anciens. Cette faible reprise de la fragmentation mécanique et de l'accumulation grossière est principalement due à la diminution de la couverture des sols par la végétation liée à l'accroissement de l'aridité. La phase humide datée vers $3300-3000$ BP n'est pas perceptible dans les Nemencha, pas plus que la phase aride vers $2900-2500$ BP (Ballais et al., 2003). L'Holocène récent historique commence avec l'épisode humide de 2400 à $2200 \mathrm{BP}$ contemporain des royaumes numides : à altitude moyenne, le dépôt fin supérieur de la basse terrasse alluviale s'accumule. Le maximum d'humidité peut avoir été atteint avec le développement d'un sol au sommet de la 
terrasse alluviale de l'oued Regada, vers $2590+/-90$ BP et au sommet de la terrasse alluviale de l'oued Chéria-Mezerra vers $2270+$ /- BP. L'accumulation de la très basse terrasse alluviale historique principale lui succède. En général, le long des oueds principaux, cette terrasse alluviale s'emboîte ou s'étage dans la précédente, consécutivement à une entaille. Dans quelques cas, peu fréquents, le dépôt alluvial s'accumule directement sur le dépôt alluvial holocène préhistorique, par exemple le long de l'oued Chéria-Mezeraa, comblant d'anciens canaux d'irrigation et daté de 1350 +/- 70 BP. Il s'agit de quelques décimètres de limons caillouteux provenant d'une phase généralisée de réorganisation de la surface qui affecte les sites préhistoriques et les couvertures de glacis, de même que les sols de la période romaine, comme celui daté de 1730 +/- $185 \mathrm{BP}$. L'entaille généralisée de la très basse terrasse alluviale se fait à une vitesse considérable (3,2 mm/an au Maghreb oriental (Ballais 1991)) et continue actuellement le plus souvent. Sa généralisation et sa vigueur montrent que l'érosion des sols a limité considérablement, voire interdit, l'infiltration des pluies intenses, le taux de ruissellement a donc augmenté et les cours d'eau ont utilisé toute leur puissance pour creuser. Dans les Nemencha, elle n'est pas interrompue par l'accumulation de la très basse terrasse alluviale post-islamique, sauf sur l'oued ChériaMezeraa, et s'accompagne de la construction de lits majeurs.

\section{La morphogénèse actuelle}

27 Elle poursuit l'évolution historique caractérisée par l'entaille vigoureuse des formations meubles privées de la protection d'une couverture végétale dense. Elle peut atteindre jusqu'à $10 \mathrm{~m}$ en contrebas des écoulements antiques et pourrait avoir été accélérée par la fixation progressive de la population nomade au $\mathrm{XX}^{\mathrm{e}}$ siècle (Chabin 1982). Sur le piémont sud, la comparaison entre la cartographie des épandages antiques effectuée par Birebent (1964), des travaux du Senatus Consulte, établi en 1890, et de la situation en 1985 (Côte 1988), montre la migration des zones d'épandage vers l'aval. A l'époque antique, elles étaient localisées au débouché même des foum, avec des systèmes de canaux divergents. À l'époque du Senatus Consulte, les aires d'épandage étaient situées de 5 à $10 \mathrm{~km}$ plus au sud, sur la partie médiane du glacis, en pente très faible. En 1985, les aires d'épandage avaient migré d'environ $2 \mathrm{~km}$ par rapport au XIX siècle, migrations confirmées par les habitants, qui l'attribuent à l'approfondissement de l'oued au débouché sur le glacis (5 à 10 mètres suivant les oueds). Cet approfondissement est à mettre en relation avec la dégradation des écosystèmes dans le haut pays Nemencha (Côte 1988).

\section{Géographie humaine}

En géographie humaine également, les Nemencha ont été peu étudiées, en particulier par les différentes géographies universelles. C'est ainsi qu'elles ne figurent même pas sur une carte économique de l'Algérie du premier quart du XXe siècle (Granger 1922, p. 91), sont limitées à huit lignes sous la plume d'A. Bernard (1937) et ne sont pas nommées par Gautier (1939), à peine par Mutin et Durand-Dastès (1995, p. 101) et limitées à une localisation cartographique par Prenant (1996, p. 765).

Par exception, l'entre deux guerres est mieux connu grâce à l'étude de Meuleman (1991). Les Nemencha sont alors peu peuplées : $82043 \mathrm{~h}$ en 1931 pour les communes de 
plein exercice et mixte de Khenchela se répartissant ainsi dans les douars : Mahmel : 8447 habitants dont 4 Français, Meggada : 6 211, Tamarout: 6 254, Aliennas : 6023 , Khanga Sidi Nadji : 2134 dont 4 Français, Ouldja-Chechar : 3 408, Taberdga : 5943 (p. 281-283).

Meuleman note les premiers conflits pour l'eau de l'oued el-Arab entre la palmeraie ancienne (XVI ${ }^{e}$ ou XVII ${ }^{e}$ siècle) de Khanga Sidi Nadji (où les confréries religieuses sont toujours actives) et les plus récentes, créées à l'amont depuis environ 1870, ou les cultures de céréales de la tribu des Zab-Chergui, conflits traités par une commission de droit hydraulique à partir de 1928. Surtout, il montre les étapes de la sédentarisation progressive des nomades : en 1911, les nomades dominaient encore dans l'est et le sud de la commune mixte de Khenchela. Dans la tribu du djebel Chechar, seuls les pauvres ne pratiquaient pas l'élevage, et celui qui n'avait pas un troupeau de l'ordre de 200 ovins, était considéré comme un indigent. L'exploitation de l'alfa fournissait un complément de revenu pour les éleveurs. Il existait seulement quelques jardins, notamment d'arbres fruitiers, dont des oliviers. Presque toute la population était (semi)-nomade et habitait des tentes. Seuls quelques artisans et des vieillards étaient sédentaires. En général donc, l'habitat sédentaire était lié à la pauvreté (p. 80-82). Il y avait, cependant, et déjà avant, plusieurs familles qui habitaient pendant une partie de l'année un gourbi, ou peut-être une espèce de maison simple, à Taberdga, et se déplaçaient pendant une autre partie de l'année avec le bétail, en habitant des tentes. À Taberdga, ces familles semi-sédentarisées possédaient des exploitations agricoles où étaient, dans plusieurs cas, employés des khammès et des «meghatta » (des employés qui recevaient une rémunération fixe). Vers 1914, cette transition du nomadisme à la vie sédentaire était déjà bien commencée. La première habitation fixe dans la dépression où se situe le centre actuel de Taberdga a été construite en 1870, mais sur la colline qui était l'ancien centre de Taberdga, des habitations ont été construites bien avant. L'abandon définitif de la tente par ces familles qui combinaient l'habitat fixe et l'habitat mobile, est intervenu plus tard (on connaît un cas d'abandon en 1939, un autre en 1947 ou 1948). Dans la région de Babar, dans le nord du territoire des Ouled Rechaich, les premiers gourbis ont été construits en 1926-1927, les premières maisons à partir de 1938-1939. Jusque dans les années 1990 au moins, on y observait des familles qui habitaient en été une maison, mais se déplaçaient en hiver avec une tente au sud.

31 Cette fixation progressive des nomades eut pour conséquence le développement de labours illicites sur les terrains de parcours, source de conflits à partir de 1927 avec la création de taxes pour les étrangers à la commune qui pratiquaient encore l'achaba dans les douars Mahmel, Meggada et Tamarout., et le recrutement $(1937,1938)$ de gardes chargés de la surveillance des communaux contre le braconnage intensivement pratiqué et pour la surveillance de la transhumance

Le commerce traditionnel a souffert de mesures administratives (Meuleman 1991, p. 170-171). La plus importante mesure de ce genre pour le Constantinois a été le décret du 24 juillet 1890 instituant, entre l'Algérie et la Tunisie, une zone où certains produits n'étaient admis à circuler qu'accompagnés soit d'une quittance de taxes, soit d'une caution, soit d'un passavant de circulation. La mesure devait permettre de surveiller l'importation et l'exportation de marchandises susceptibles d'alimenter la contrebande. La zone en cause était très étendue : elle était délimitée d'un côté par la frontière algéro-tunisienne, de l'autre par une ligne partant de La Calle pour aboutir à Touggourt, en passant pas Souk-Ahras, Bordj Meskiana, Khenchela, la route de 
Khenchela à Batna jusqu'à l'Oued Firès, El-Kantara et Chegga. Pour les matières concernées (entre autres le sucre, le café, le thé, le coton en grosse quantité), il fallait solliciter un permis de circulation auprès du Receveur des Douanes.

L'activité du bâtiment était très réduite. Lorsque, au début de l'année 1932, on cherchait des maçons pour construire un logement pour l'Administrateur adjoint à Khanga Sidi Nadji, on ne trouva personne sur place : par suite de la mauvaise situation économique, tous les maçons locaux avaient quitté la ville.

\section{BIBLIOGRAPHIE}

AMIA Z., KASSAB M., ISSAD M., 2005 - « Potentialités pétrolières et applications de la stratigraphie séquentielle sur les réservoirs crétacés de la bordure sud du bassin sud-est constantinois », Colloque Géologie du Grand Constantinois et des confins algéro-tunisiens, Constantine, Recueil des résumés, p. 2-3.

BAGHLOUL M., 1974 - Géologie de la région des dômes (Algérie orientale), thèse $3^{\mathrm{e}}$ cycle, Paris VI.

BALLAIS J.-L., 1976 - « Morphogénèse holocène dans la région de Chéria (Nementchas-Algérie) », Actes Symp. versants en Pays médit., Aix-en-Provence, 1975, CEGERM, vol. V, p. 127-130.

BALLAIS J.-L., 1981 - Recherches géomorphologiques dans les Aurès (Algérie), thèse Etat, Paris I, 566 p. + 107 photos hors-texte.

BALLAIS J.-L., 1989 - « Aurès », Encyclopédie Berbère, Aix-en-Provence, VII, p. 1066-1095.

BALLAIS J.-L., 1991 - « Vitesses d'accumulation et d'entaille des terrasse alluviales holocènes et historiques au Maghreb oriental », Physio-Géo, Paris, n² 22-23, p. 89-94.

BALLAIS J.-L., 1995 - « Alluvial Holocene terraces in eastern Maghreb : Climate and anthropogenic controls ", in J. LEWIN, M. M. MACKLIN, J. C. WOODWARD, eds, Mediterranean Quaternary River Environments, A.A. Balkema, Rotterdam, p. 183-194.

BALLAIS J.-L., BENAZzouz M. T., 1994 - « Données nouvelles sur la morphogenèse et les paléoenvironnements tardiglaciaires et holocènes dans la vallée de l'oued Chéria-Mezeraa (Nemencha, Algérie orientale) ", Méditerranée, $\mathrm{n}^{\circ}$ 3.4, p. 59-71.

BALLAIS J.-L., BENAZZOUZ M. T., BENMOHAMMADI A., 2003 - « Environmental dynamics and land occupation in the Saharan margins of the Holocene Maghreb ", in E. FOUACHE éd. The Mediterranean World. Environment and History, Paris, Elsevier, p. 341-351.

BENAZZOUZ M.T., 2000 - Morphogenèse éolienne holocène et actuelle dans l'Atlas saharien oriental (Algérie) : conséquences sur la désertification, thèse d'État, Constantine.

BERGHEUL M., 2005) - « Géologie et exploitation pétrolière de l'Atlas saharien oriental et des confins algéro-tunisiens », Colloque Géologie du Grattd Constantinois et des confins algéro-tunisiens, Constantine, Recueil des résumés, p. 7.

BERNARD A., 1937 - « Afrique septentrionale et occidentale », Géographie Universelle, P. VIDAL DE LA BLACHE et L. GALloIS dir., Armand Colin, Paris, 284 p. 
BIREBENT J., 1964 - Aquae Romanae, Thèse Université, Alger, 523 p.

BIROT P., DRESCH J., 1953 - La Méditerranée et le Moyen-Orient, PUF, Paris, t. I, 552 p.

BLAYAC J., 1899 - « Le pays des Nemenchas à l'est des monts Aurès », Annales de Géographie, 8, 38, p. $141-159$.

CHABIN J.-P., 1982 - L'Homme et le milieu naturel à l'époque historique contemporaine (1850-1980) sur les confins sahariens de l'est algérien : région des Nemencha, communes de El-Ma-El-Abiod et Bir-El-Ater, thèse de 3e cycle, Dijon, 2 t., 519 p. + Cartes et diagrammes HT.

CHABIN J.-P., 1988 - «Climatologie et géographie. Exemple d'une région présaharienne de l'Est algérien », Climats et Climatologie, volume d'hommage offert au professeur Pierre PAGNEY, Université de Bourgogne, p. 63-77.

CÔTE M., 1988 - « Géomorphologie et évolution historique sur quelques piémonts de l'Est algérien », Etudes Méditerranéennes, 12, p. 221-227.

COUVERT M., 1972 - «Variations paléoclimatiques en Algérie », Libyca, 20, p. 45-48.

DESPOIS J., RAYNAL R., 1967 - Géographie de l'Afrique du Nord-Ouest, Payot, Paris, 570 p.

DJEBAÏLI S., 1984 - Steppe algérienne. Phytosociologie et Ecologie, OPU, Alger, 177 p., il manque les pages 31 à 62) + volume de figures hors-texte.

ENNERY, HIRTH, 1840 - Dictionnaire général de Géographie universelle ancienne et moderne, Strasbourg, Baquol et Simon, t. troisième, $818 \mathrm{p}$.

FARRAND W.R., STEARNS C.H., JACKSON H.E., 1982 - « Environmental setting of Capsian and related occupations in the high plains of Northeastern Algeria », Geol. Soc. of America, 14, 7, p. 487.

GAUTIER E.-F., 1939 - L'Afrique blanche, Paris, Arthème Fayard, 366 p.

GRANGER E., 1922 - Nouvelle Géographie Universelle, Paris, Hachette, t. II, 496 p.

IDRISî (1999). - La première géographie de l'Occident, Paris, GF Flammarion, 516 p.

KADDACHE M., 1992 - L’Algérie durant la période ottomane, réimpression, Alger, OPU, 239 p.

KALLA M., 1995 - Recherches géomorphologiques dans le massif des Nemenchas (Algérie), thèse, Université Paris VII, 380 p., cartes et photos hors-texte.

KALLA-DRIDI H., 1994 - Recherches Géomorphologiques dans la plaine de Tebessa et ses bordures (Algérie Orientale), thèse Paris I, 290 p., 3 planches hors-texte.

LAFITTE R., 1939 - Étude géologique de l'Aurès, Bull. Serv. Carte géol. Alg., $2^{\text {e }}$ série, n 15, 484 p., XXXIV pl. h.-t., 10 pl. ph. h.-t., 1 carte géologique h.-t.

LE HOUÉROU H.N., CLAUdiN J., POUCET M., 1979 - «Étude bioclimatique des steppes algériennes », Bulletin de la Société d'Histoire Naturelle de l'Afrique du Nord, t. 68, fasc. 3 et 4, p. 33-74.

LUBELL D., BALLAIS J.-L., GAUTIER A., HASSAN F.A., 1975 - « The prehistoric cultural ecology of Capsian escargotières ", Libyca, XXIII, p. 43-121.

MASQUERAY É., 1878 - « Le Djebel Chechar », Revue Africaine, vol. 22, p. 26-48, 129-144, 202-213, 259-281 (appendice).

MEULEMAN J.H., 1991 - Le Constantinois entre les deux guerres mondiales, OPU, Alger, 388 p. MUTIN G., DURAND-DASTÈS F., 1995 - « Afrique du Nord, Moyen-Orient, Monde indien », Géographie Universelle R. BRUNET dir., Paris, Belin/Reclus, 480 p. 
PRENANT A., 1996 - Algérie, Encyclopedia Universalis, t. 1, p. 763-766.

RECLUS É., 1886 - « L'Afrique septentrionale », Nouvelle Géographie Universelle, t. XI, Paris, Hachette, $919 \mathrm{p}$.

SHAW Dr., 1980 - Voyage dans la régence d'Alger ; trad. J. Mac Carthy, $2^{\mathrm{e}}$ édit., Tunis, Bouslama, 402 p.

INDEX

Mots-clés : Algérie, Aurès, Economie, Géographie 